\title{
Hafnium and Erbium Codoped ZnO Thin Films as Anode Material and Spectral Modificater for Solar Cells Applications
}

\author{
Z. Makir $^{1,2} \cdot$ Oumar A. Niasse $^{1} \cdot$ I. El Ouedghiri ${ }^{2} \cdot$ F. Dia ${ }^{1} \cdot$ N. Mbengue ${ }^{1} \cdot$ Z. Sofiani $^{2}$ \\ ${ }^{1}$ Laboratory of Semiconductors and Solar Energy, Dept of Physics, Faculty of Sciences, University Cheikh Anta Diop of Dakar, \\ Sénégal. \\ ${ }^{2}$ LAMPCER Lab., Phys. Dept., Faculty of Sciences and Technologies of Mohammedia, University Hassan II of Casablanca, \\ Morocco.
}

\section{ABSTRACT}

The spectral mismatch between solar cells and incident radiation is a fundamental factor limiting their efficiencies. There are materials and luminescent processes which can modify the incident sunlight's properties to better suit the cell's optimal absorption regions. Developing new structures or optical devices to improve the trapping of light within solar cells is a real challenge. This work is part of the theme of increasing the efficiency of solar cells by relying on techniques offered by optical engineering. Our aim is to study the visible photoluminescence spectra after Ultraviolet (UV) excitation, of hafnium and erbium codoped zinc oxide, in order to modify the solar spectrum and adapt it to the solar cell, and consequently to enhance its efficiency. In addition we will study the use of these films as photovoltaic anode. (C) $2021 \cdot$ INScienceIN. All rights reserved

ARTICLE HISTORY

Received 03-05-2021

Revised 26-05-2021

Accepted 26-04-2021

Published 16-06-2021

\section{KEYWORDS}

Photoluminescence

Thin films

$\mathrm{ZnO}$

Rare earth

\section{Introduction}

Thin films based on transparent conductive oxides (TCO) have been widely studied due to their high optical transmission, electrical conduction, and potential applications in photoelectronic devices such as displays and solar cells [1-2]. The most marketed material (TCO) is indium tin oxide (ITO). It has been widely used in flat panel displays including liquid crystal displays, plasma displays and organic electro-luminescent displays (OLEDs) [3-4] and organic photovoltaics [5]. However, the growth in production of these optoelectronic devices has led to double the demand for the ITO material, and the shortage of indium resources worries these industries. Thus, there remains an urgent need for the development of an effective solution for the search for alternatives to ITO at low cost. Doped zinc oxide $(\mathrm{ZnO})$ is a possible alternative to ITO because of its optical and electrical characteristics, nontoxicity, low price and abundance. Consequently, the thin films of doped $\mathrm{ZnO}$ have a good potential for optoelectronic applications in particular in photovoltaics where it could be used as an anode [6] or as a down conversion conch [7-8]. $\mathrm{ZnO}$ is known by its wide band gap of about $3.33 \mathrm{eV}$ and its exciton binding energy ( $60 \mathrm{meV})$. It is considered to be an excellent near ultraviolet emitter at room temperature. It is also a semiconductor material having a low electrical resistivity in most cases where it is of type n, with high transmission in the visible and near infrared. In addition, its physicochemical properties in the form of a thin film are directly related to the structure, morphology, size and intrinsic (such as oxygen vacancies and $\mathrm{Zn}$ interstitials) or extrinsic (dopants) defects in crystallites of $\mathrm{ZnO}$.

The current interest of the scientific community in this TCO is based on improving its various properties in order to improve its performance and accelerate the race towards new applications. Indeed, the incorporation of impurities into the $\mathrm{ZnO}$ matrix can considerably modify its properties.
Generally, ZnO thin films are doped with trivalent cations or with appropriate anions to improve the electrical conductivity of the material. In our work we have been interested in doping with rare earth ions. Nowadays, the doping of $\mathrm{ZnO}$ thin films with lanthanides called "rare earths" has attracted a lot of attention in several fields due to their electronic and optical properties. In principle, rare earth ions exhibit $4 \mathrm{f}-4 \mathrm{f}$ optical transitions in a wide range of wavelengths from ultra-violet to infrared, thus possessing numerous very intense luminescent bands, which are used. in various applications. The problem with rare earths is that increasing the concentration of rare earth ions leads to a decrease in luminescence. To overcome this problem, it is in our interest to dope with a coactivator such as aluminum for example. Several studies have noted the dispersing effect of aluminum co-doping on rare earth (RE) ions incorporated in glasses or silica gels [9-10]. The details of the ion isolation process are not clear to us. However, it is generally argued that the rare earth oxides being poorly soluble in silica form aggregates, the addition of aluminum (Al) increases this solubility. Rare earth ions preferentially bind with aluminum (Al) ions, thus forming Al-O-RE bonds rather than RE-O-RE bonds. Consequently, the space between the rare earth elements would be greater in the case of matrices containing aluminum, than in those without. In the case of $\mathrm{ZnO}$, the band-to-band excitation of $\mathrm{ZnO}$ only induces luminescence of the self-activated (SA) ZnO centers. However, adding a small amount of a coactivator [11], such as $\mathrm{Li}^{+}$ions or nitrogen induces energy transfer from the semiconductor to $\mathrm{RE}^{3+}$ ions. Then, the excitation of $\mathrm{ZnO}$ in the forbidden band can lead to the luminescence of $\mathrm{RE}^{3+}$ ions. This characteristic is required for possible applications as a converting layer for photovoltaics. This is widely used for adapting the solar spectrum to the absorption band of the active material in photovoltaics. Indeed, the main limitation 
of the efficiency of photovoltaic cells comes from the polychromatic nature of the solar spectrum. Thus, potentially, if we transform the wavelength of the incident photons to bring it back to that corresponding to the air gap of the semiconductor, the cell could achieve a very high efficiency.

The aim of this article is to demonstrate the effect of hafnium as coactivators in order to improve the photoluminescence of erbium ions. To our knowledge, this article is the first report on the use of hafnium as coactivators in the erbium-doped $\mathrm{ZnO}$ matrix. This in order to consider an integration of a thin layer of $\mathrm{ZnO}$ in photovoltaic solar cells, a systematic study of the influence of the doping of $\mathrm{ZnO}$ on their properties remains a necessary step for the optimization of their performance in order to use them as transparent and conductive electrode [12] and / or converting layer [13]. The low conversion efficiency of solar cells is mainly due to the shift between the solar spectrum and the spectral response of PV solar cells, therefore due to poor exploitation of the solar spectrum. As example, for crystalline silicon (c-Si) solar cells which largely dominate the current market, two intrinsic spectral limitations exist. The limitation in the infrared $(\lambda>1100 \mathrm{~nm})$ is due to the gap of silicon which cannot absorb lower energies. With regard to high energy radiation (UV and blue wavelength), the conversion limitation comes from the thermalisation of charge carriers and the losses induced by the excitation of an Auger electron. The advantage of wavelength conversion materials is that they can be integrated fairly easily into the cells without modification of their active part. We make just front panel for down-conversion (DC) systems and rear panel for up-conversion (UC) systems, as shown in Fig. 1.
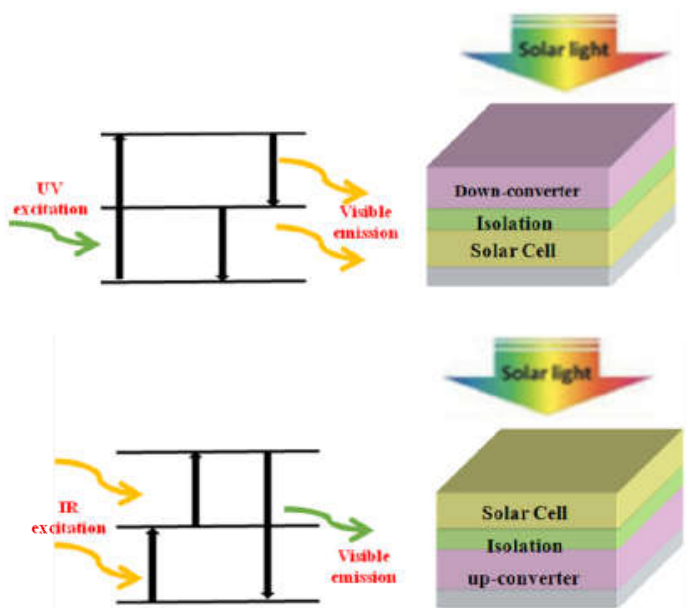

Figure 1: Principle of spectrum modification by down- and upconversion

Materials that can act as down or up converters can reduce the cost of fabrication of these systems. J. Day et al. gave an estimate of the maximum mass of rare earth ions that can be added per $\mathrm{W}$ of solar cell to reduce more the cost per $\mathrm{W}$ [14].

Thin films of zinc oxide are made using a wide variety of techniques due to the diversity of applications of this material. They can be obtained by operating using the physical "PVD" methods, the "CVD" chemical methods, sol gel [15], electroplating by electrochemical synthesis [15] and Reactive chemical spraying in liquid phase, which We will be interested in this study, also called "spray pyrolysis" from aqueous or alcoholic solutions of a zinc salt is a technique widely used especially for oxides because the deposits are produced in a normal atmosphere [17].

\section{Experimental \\ Materials and methods}

Thin films are prepared using the spray pyrolysis technique. Thin films of zinc oxide co-doped with hafnium and erbium were prepared from a solution of zinc chloride, cerium chloride and hafnium chloride dissolved in deionized water and transported to the nozzle $(4 \mathrm{ml} / \mathrm{min}$ and distance nozzle-substrates $40.5 \mathrm{~cm}$ ), which is sprayed in fine droplets using air as carrier gas. This solution was deposited for 4 minutes on clean glass substrates heated to $450^{\circ} \mathrm{C}$. The structural analysis of the thin layers obtained was carried out by X-ray diffraction (monochromatic Cuk $\alpha$ radiation $\lambda=0.15405 \mathrm{~nm}$ ). These films were studied electrically by means of resistivity measurements by the method of Van Der Pauw. Photoluminescence measurements were made using an Omnichrome HE-Cd laser $(\lambda=325 \mathrm{~nm}, 42 \mathrm{~mW}$ ) was used to excite the sample.

\section{Results and Discussion}

After codoping, the Er and Hf doped zinc oxide layers obtained under optimal operating conditions were characterized by X-ray diffraction. This is in order to identify the phases present and the effect of the doping on the structure. Figure 2 represents the diffractograms of the various layers doped with $5 \% \mathrm{Er}$ and codoped respectively at $2 ; 5$ and $7 \% \mathrm{Hf}$.

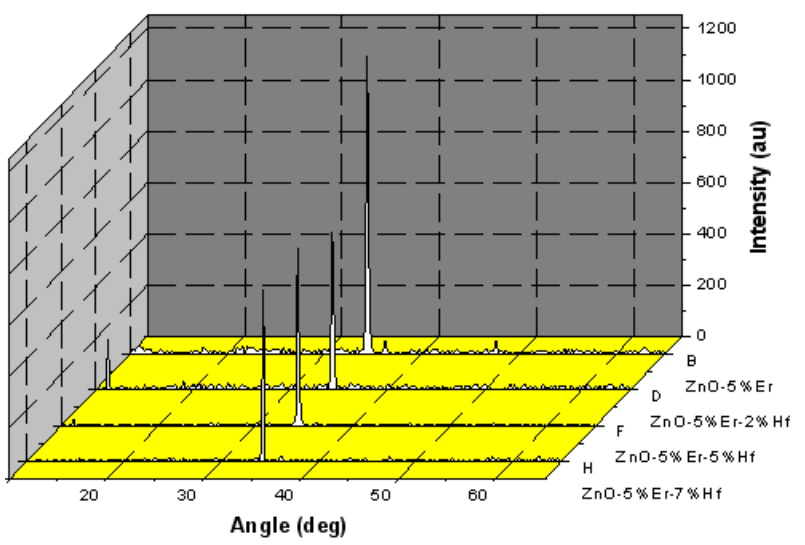

Figure 2: Diffractograms of ZnO layers doped with Er respectively at $0 ; 2 ; 5$ and $7 \%$ Hf

According to Fig. 2, all the layers are well structured. They crystallize in the hexagonal Würtzite type structure with the same preferential orientation, along the $\mathrm{c}$ axis perpendicular to the surface of the substrate. This orientation is due to the fact that the plane (002) is the densest and corresponds to the minimum energy surface [18-19]. This same observation is made on layers prepared by simultaneous evaporation of zinc and erbium oxide [20]. In our case, the contribution of hafnium is manifested by the change in the intensity of diffraction and the width at half height. We also noticed a slight change in the position of the diffraction peak which reflects the stresses to which the mesh is subjected following the insertion of the hafnium. No additional peak associated with dierbium trioxide, hafnium dioxide or any other accidental impurities were detected. This proves that the $\mathrm{Hf}^{2+}$ and $\mathrm{Er}^{3+}$ ions incorporated very well into the $\mathrm{ZnO}$ network. It is clear that the incorporation of $\mathrm{Hf}^{2+}$ and $\mathrm{Er}^{3+}$ ions, with an 
ionic radius ( 0.81 and $0.89 \AA$ respectively) greater than that of $\mathrm{Zn}(0.74 \AA)$ in the $\mathrm{ZnO}$ matrix deteriorated the crystallinity of the film.

Table 1: Lattice parameters of Er doped and Hf codoped ZnO thin

\begin{tabular}{cccc}
\multicolumn{3}{c}{ films } \\
\hline Sample & $\mathrm{c}(\AA)$ & $\mathrm{a}(\AA)$ & Volume $\left(\AA^{3}\right)$ \\
\hline Zn0: $5 \% \mathrm{Er}$ & 5.201 & 3,245 & 47.429 \\
Zn0: $5 \% \mathrm{Er}: 2 \% \mathrm{Hf}$ & 5.203 & 3.251 & 47.623 \\
Zn0: $5 \% \mathrm{Er}: 5 \% \mathrm{Hf}$ & 5.206 & 3.255 & 47,768 \\
Zn0: $5 \%$ Er : $7 \% \mathrm{Hf}$ & 5.211 & 3.259 & 47.931 \\
\hline
\end{tabular}

The value $\mathrm{c}$ of the lattice parameter of the $\mathrm{Er}$ and $\mathrm{Hf}$ codoped $\mathrm{ZnO}$ thin films were calculated from the X-ray diffraction pattern. From the calculated value of the lattice parameters (Table 1), we find that these parameters increase with the percentage of hafnium (Hf) concentration from $0 \%$ to $7 \% \mathrm{Hf}$ in the $\mathrm{ZnO}$ network. This can be explained by the cationic substitution of $\mathrm{Zn}^{2+}$ ions (ionic radius $0.74 \AA$ ) by the $\mathrm{Er}^{3+}$ and $\mathrm{Hf}^{2+}$ ions $(0.81$ and $0.89 \AA$ respectively).

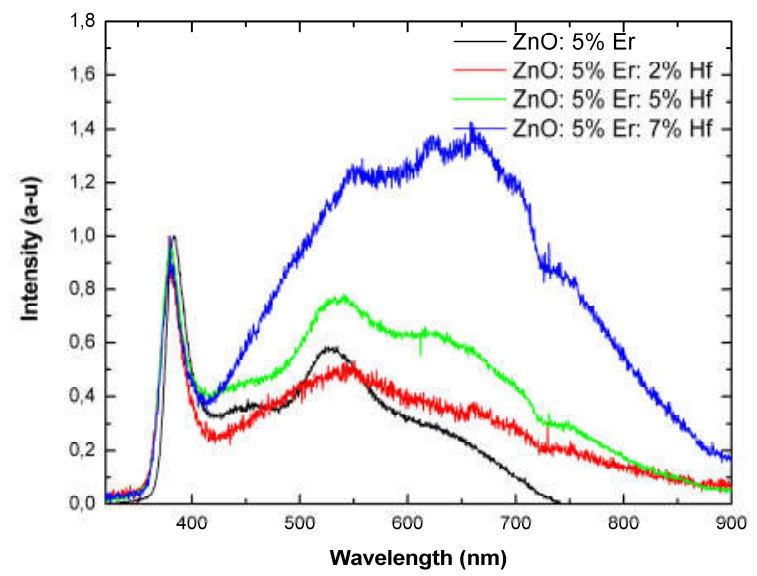

Figure 3: Photoluminescence spectra of films of $\mathrm{ZnO}$ layers doped with Er respectively at 0; 2; 5 and $7 \% \mathrm{Hf}$

The study of the luminescence of $\mathrm{ZnO}$ has revealed interesting characteristics of this material. Figure 3 represents the photoluminescence spectrum of $\mathrm{ZnO}$ doped with erbium and hafnium ions. For a better evaluation of the doping effect, the photoluminescence spectra were normalized against the UV band. This figure shows that these films are luminescent at three wavelengths, characteristic of $\mathrm{ZnO}$, at 380,530 and $675 \mathrm{~nm}$ corresponding to luminescence in the ultraviolet, bluegreen and in the red. The first band located at the wavelength of $380 \mathrm{~nm}$, associated with the band-to-band transition, is the origin of the intrinsic properties of $\mathrm{ZnO}$. The presence of this band indicates the good crystalline quality observed by the X-ray diffraction. The second band located at $530 \mathrm{~nm}$ is a characteristic of the zinc oxide of the blue green emission. The center responsible for this emission in thin films of $\mathrm{ZnO}$ has been studied by several authors. Minami et al. [21] attributed this emission to crystal defects. Indeed, the emission in this area is associated with a transition of the self-activation centers formed by a doubly ionized zinc vacancies $\mathrm{V}_{\mathrm{Zn}}^{\prime \prime}$ and an ionized interstitial site $\mathrm{Zn}^{+}$. Other authors have reported that this emission is due to a radiative transition between oxygen donor vacancies and zinc acceptor vacancies [2223]. While, T. Sekiguchi et al. have suggested that this luminescence may also be due to copper atoms which become incorporated into the layer during growth [24].

The last band centered around $675 \mathrm{~nm}$ is a characteristic of the emission in the red. Indeed, the emission in the red can have different origins: electron-phonon coupling, distortion of the network, localization of charge carriers due to the interface effect. Lohnert et al. [25] also noticed that the luminescence spectra observed between 450 and $700 \mathrm{~nm}$ depend on the partial pressure of oxygen. This led us to suggest that the emission at $675 \mathrm{~nm}$ in the red of these layers can be attributed to the transition from a neutral oxygen atom $V_{0} x$ to a simply ionized oxygen $V_{0}$.

In the layers codoped by erbium and hafnium ions, in addition to the characteristic bands of $\mathrm{ZnO}$ we also observe emissions from the erbium ion. A simple comparison between the spectrum of the Erbium doped layer and the Erbium and Hafnium codoped layers also shows a clear improvement in the luminescence intensity. We have noticed the appearance generation of new bands. We can attribute the visible luminescence lines to the transitions between the $4 \mathrm{f}$ levels of the Er ion ${ }^{3+}$. They correspond to the transitions around $440 \mathrm{~nm}, 450 \mathrm{~nm}, 488 \mathrm{~nm}, 537,559 \mathrm{~nm}$ and $653 \mathrm{~nm}$ assigned respectively to ${ }^{4} \mathrm{~F}_{3 / 2} \rightarrow{ }^{4} \mathrm{I}_{15 / 2},{ }^{4} \mathrm{~F}_{5 / 2}$ $\rightarrow{ }^{4} \mathrm{I}_{15 / 2},{ }^{4} \mathrm{~F}_{7 / 2} \rightarrow{ }^{4} \mathrm{I}_{15 / 2},{ }^{2} \mathrm{H}_{11 / 2} \rightarrow{ }^{4} \mathrm{I}_{15 / 2},{ }^{4} \mathrm{~S}_{3 / 2} \rightarrow{ }^{4} \mathrm{I}_{15 / 2}$ et ${ }^{4} \mathrm{~F}_{9 / 2}$ $\rightarrow{ }^{4} \mathrm{I}_{15 / 2}$. Emissions are not very well resolved because of peak broadening which is probably related to electron phonon interactions [26]. This is a characteristic of disordered media with a gap between $(1.5-3.5 \mathrm{eV})$, in particular by erbium doping [27]. This observed change can be explained by a deactivation phenomenon linked to the occupation of vacant zinc sites by erbium ions [28]. This promotes energy transfer from the $\mathrm{ZnO}$ matrix to the erbium ions. This is because hafnium has a dispersive effect on erbium ions by reducing erbium aggregates. Hence, the erbium ions find an environment more conducive to their incorporation into the material. Thus, we have shown that the increase in the codoping rate leads on the one hand to the appearance of new luminescence bands, on the other hand to the intensification of all the transitions. This is a sign that the hafnium codoping facilitated the insertion of erbium into the zinc oxide matrix thereby promoting internal erbium transitions. In addition to this improvement, we also notice that our materials convert UV light, Excitation, into a wide band in the visible area which corresponds to the most absorbed band by photovoltaic cells in terms of dyes and organics. This makes this sample a good candidate for the down conversion layer used to match.

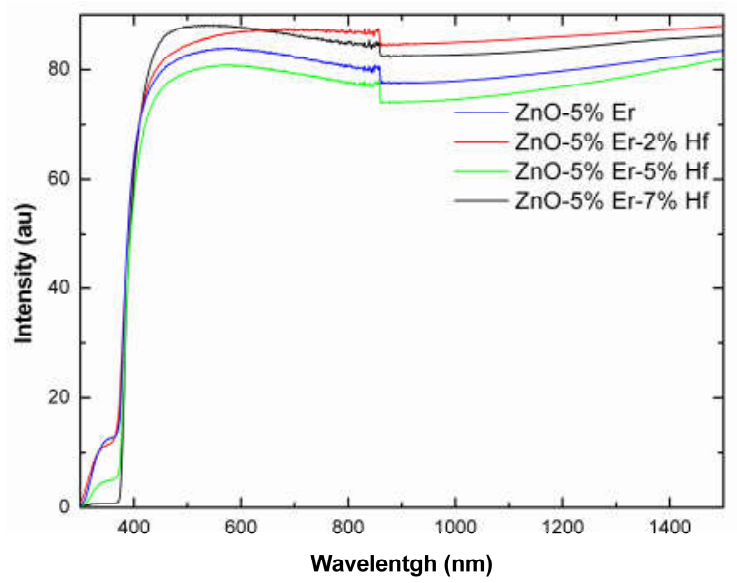

Figure 4: Transmission spectra of films of Er-doped $\mathrm{ZnO}$ layers respectively at $0 ; 2 ; 5$ and $7 \% \mathrm{Hf}$ 
The majority of optoelectronic devices require transparent layers in the visible range. In this approach, we studied the optical transmission of the layers developed according to the composition. Figure 4 shows the optical transmission spectra of the layers differently codoped $5 \%$ Er and 2, 5, $7 \%$ Hf. It can be seen that the spectra are composed of three regions: A region of high transparency, located between 400 and $800 \mathrm{~nm}$; the value of the transmission varies between $80 \%$ and $95 \%$. This value gives the thin layers of undoped and doped $\mathrm{In}_{2} \mathrm{O}_{3}$ the character of transparency in the visible range. In this wavelength range, interference fringes are observed in the films produced. These fringes, characterized by the undulations of the curves, are due to the multiple reflection of the radiation on the two interfaces of the film. This result indicates that the films prepared under these conditions are homogeneous and have more smooth surfaces. The second region is characterized by a slight reflection in the infrared domain. This phenomenon is generally attributed to absorption by free carriers, given their high concentration in these films. The third region shows strong absorption. The latter corresponds to the fundamental absorption $(\lambda<400 \mathrm{~nm})$ of films. This absorption is due to the inter-band electronic transition.

Table 2: Gap energies of Er doped and Hf codoped ZnO thin films

\begin{tabular}{ccccc}
\hline \multirow{2}{*}{$\mathrm{ZnO}-\mathrm{Hf}$} & $\begin{array}{c}\mathrm{ZnO}- \\
5 \% \mathrm{Er}\end{array}$ & $\begin{array}{c}\text { ZnO-5\% } \\
\mathrm{Er}-2 \% \mathrm{Hf}\end{array}$ & $\begin{array}{c}\text { Zn0-5\% Er- } \\
5 \% \mathrm{Hf}\end{array}$ & $\begin{array}{c}\text { ZnO-5\% Er- } \\
7 \% \mathrm{Hf}\end{array}$ \\
\hline $\mathrm{Eg}(\mathrm{eV})$ & 3,888 & 3.816 & 3,796 & 3.218 \\
\hline
\end{tabular}

Table 2 present the variation of gap energies of Er doped and $\mathrm{Hf}$ codoped $\mathrm{ZnO}$ thin films. This variation is probably due to the formation of localized states at the end of the band and in the forbidden band. Erbium and hafnium codoping causes the inter-atomic distances to vary, thus creating a disorder, leading to the disappearance of the band edges which were described in the case of crystal lattices and which were delimited by the valence and conduction energies. However, we observe the creation of states located in the tail of bands at the borders of the forbidden band, in the valence and conduction band. These are responsible for reducing the gap energies.

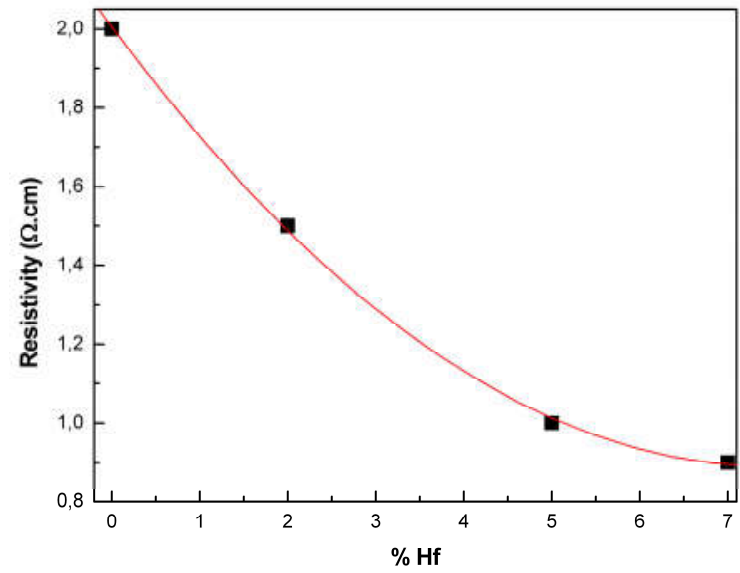

Figure 5: Evolution of the resistivity of $\mathrm{ZnO}$ thin films doped with Errespectively at $0 ; 2 ; 5$ and $7 \% \mathrm{Hf}$

The electrical properties of a thin layer of $\mathrm{ZnO}$ will directly influence the performance of the device in which this layer will be integrated. This is why we have studied the electrical behavior of thin films by determining their electrical resistivity as a function of the co-dopant rate as shown in Fig. 5. The electrical measurements that we carried out are obtained using the four-point method. The influence of the doping rate on the electrical resistivity $(\rho)$ of thin films of $\mathrm{ZnO}$ codoped by erbium and hafnium. This codoping decreases the value of the electrical resistivity to the minimum value of $9.10^{-2} \Omega . \mathrm{cm}$ and beyond this doping rate, it remains practically constant. This decrease in electrical resistivity with the doping rate can be interpreted by the increase in the number of charge carriers (electrons) originating from the $\mathrm{Hf}^{4+}$ donor ions which are incorporated in the substitutional or interstitial locations of the $\mathrm{Zn}^{2+}$ cations. Each substitution of $\mathrm{Zn}$ by Hf gives two free electrons which will contribute to the electrical conduction. From the results of transmission and resistivity, we can conclude that our materials, especially with codoping, could play the role of a photovoltaic anode [29-31].

\section{Conclusions}

Thin films of Hafnium and Erbium codoped Zinc Oxide have been prepared using spray pyrolysis techniques. A systematic study according to codoping rate, the crystallinity revealed that the codoped zinc oxide layers have good adhesion to the substrates and crystallize in the same hexagonal structure of $\mathrm{ZnO}$ undoped with an orientation of the crystallites in the direction (002). Transmission of the order of $90 \%$ and $85 \%$ in the visible range, accompanied by a decrease of resistivity as function as the codoping rate. This justifies the use of our thin films as an anode for photovoltaics. Finally, the photoluminescence, after UV excitation, exhibits a large and important luminescence in the visible, which could be used as a down converter thin film in order to adapt the solar spectrum to the absorption of active materials in photovoltaics.

\section{Conflicts of Interest:}

In view of this submission the authors declare that there are no conflicts of interest.

\section{References}

1. KL. Chopra, S. Major and DK. Pandya DK, Transparent conductors, Thin Solid Films, 1983102 1, 1-46.

2. LT. Yin, JC. Chou, WY. Chung, TP. Sun and SK. Hsiung, Separate structure extended gate $\mathrm{H}+$-ion sensitive field effect transistor on a glass substrate, Sensors Actuators B, $2000,71,106$

3. BH. Lee, IG. Kim, SW. Cho and SH. Lee, Effect of process parameters on the characteristics of indium tin oxide thin film for flat panel display application, Thin Solid Films, 1997, 30225.

4. JS. Kim, M. Granstrom, RH. Friend, N. Johansson, WR. Salaneck, R. Daik, WJ. Feast and F. Cacialli, Indium-tin oxide treatments for single- and double-layer polymeric lightemitting diodes: The relation between the anode physical, chemical, and morphological properties and the device performance, J. Appl. Phys, 1998, 84, 6859.

5. F. Mohtaram, S. Borhani, M. Ahmadpour, P. Fojan, A. Behjat, HG. Rubahn, M. Madsen, Electrospun ZnO nanofiber interlayers for enhanced performance of organic photovoltaic devices, Solar Energy, 2020,197, 311-316.

6. O Adedokun, IT Bello, YK Sanusi, AO Awodugba, Effect of precipitating agents on the performance of $\mathrm{ZnO}$ nanoparticles based photo-anodes in dye-sensitized solar cells, Surfaces and Interfaces, 2020, 21, 100656.

7. V. Kumar, OM. Ntwaeaborwa, T. Soga, V. Dutta and HC Swart, Rare Earth Doped Zinc Oxide Nanophosphor Powder: A Future Material for Solid State Lighting and Solar Cells, ACS Photonics 2017, 4, 2613-2637. 
8. M. El Jouad, E. M Bouabdalli, S. Touhtouh, M. Addou, N. Ollier and B. Sahraoui, Red luminescence and UV light generation of europium doped zinc oxide thin films for optoelectronic applications, Eur. Phys. J. Appl. Phys, 2020, 91, 10501.

9. J. Wang, WS Brocklesby, JR Lincoln, JE Townsend, and DN Payne, Local structures of rare-earth ions in glasses: the 'crystal-chemistry' approach, Journal of Non-Crystalline Solids, 1993, 163 (3): 261-267.

10. MJ Lochhead and KL Bray, Rare-Earth Clustering and Aluminum Codoping in Sol-Gel Silica: Investigation Using Europium(III) Fluorescence Spectroscopy, Chem. Mater., 1995, 7: 572-577.

11. W.M. Jadwisienczak, H.J. Lozykowski, A. Xu, B. Patel, Visible emission from $\mathrm{ZnO}$ doped with rare-earth ions, Journal of Elec Materi, 2002, 31, 776-784.

12. H. Park, S. Chang, M. Smith, S. Gradečak \& J. Kong, Interface engineering of graphene for universal applications as both anode and cathode in organic photovoltaics, Sci Rep, 2013, 3, 1581.

13. A. Verma, SK. Sharma, Down-conversion from Er3+Yb3+ codoped CaMoO4 phosphor: A spectral conversion to improve solar cell efficiency, Ceramics International, 2017, $43,12,8879-8885$.

14. J. Day, S. Senthilarasu, TK. Mallick, Improving spectral modification for applications in solar cells, Renewable Energy, 2019, 132, 186-205.

15. Y. Natsume, H. Sakata, Electrical and Optical Properties of Zinc Oxide Films Post-Annealed in $\mathrm{H} 2$ after Fabrication by Sol-Gel Process, Materials Chemistry \& Physics, 2002, 78, 170.

16. S. Peulon, D. Lincot, Mechanistic Study of Cathodic Electrodeposition of Zinc Oxide and Zinc Hydroxychloride Films from Oxygenated Aqueous Zinc Chloride Solutions, J. Electrchem. Soc, 1998, 145, 864.

17. R. Ayouchi, F. Martin, D. Leinen, JR Ramos-Barrado, Growth of pure $\mathrm{ZnO}$ thin films prepared by chemical spray pyrolysis on silicon, J. Cryst, Growth, 2003, 247, 497.

18. JF Chang, L. Wang, MH Hon, Studying of transparent conductive $\mathrm{ZnO}$ : Al thin films by RF reactive magnetron sputtering, J. Cryst. Growth, 2000, 211, 93.

19. B. Cheng, Y. Xiao, G. Wu, L. Zhang, Controlled Growth and Properties of One-Dimensional ZnO Nanostructures with Ce as Activator/Dopant, Adv. Funct. Mater, 2004, 9, 1.

20. XT Zhang, YC Liu, JG Ma, YM Lu, DZ Shen, W. Xu, GZ Zhong, XW Fan, Room-temperature blue luminescence from $\mathrm{ZnO}$ : Er thin films, Thin Solid Films, 2002, 413, 257-261.

21. T. Minami, H. Nanto and S. Takata, Luminescent properties of sputtered ZnO thin films, J. Lumin., 1981, 24/25, 63.

22. R. Dingle, Luminescent Transitions Associated With Divalent Copper Impurities and the Green Emission from Semiconducting Zinc Oxide, Pys. Rev. Lett, 1969, 23, 579.

23. HJ Egelhaaf, D. Oelkrug, Luminescence and nonradiative deactivation of excited states involving oxygen defect centers in polycrystalline ZnO, J. Cryst. Growth, 1996, 161, 190.

24. T. Sekiguchi, N. Ohashi, Y. Trada, Effect of Hydrogenation on ZnO Luminescence, Jpn. J. Appl. Phys, 1997, 36, L289.

25. K. Lohnert and E. Kubalek, Microscopy of semiconducting Materials, The Institute of Physics Bristol, 1983,1st edition, p. 303.

26. JL Bubendorff, J. Ebothé, A. El Hichou, R. Dounia and M. Addou, Luminescent spectroscopy and imaging of textured sprayed Er-doped ZnO films in the near ultraviolet and visible regions, J. Appl. Phys., 2006, 100, 014505.

27. IV Kityk, J. Wasylak, D. Dorosz, J. Kucharski, S. Benet, and H. Kaddouri, PbO-Bi203-Ga203-BaO glasses doped by Er3+as novel materials for IR emission, Opt. Laser Technol., 2001, $33,7,511$.

28. A. El Hichou, M. Addou, A. Bougrine, R. Dounia, J. Ebothé, M. Troyon, M. Lamrani, Cathodoluminescence properties of undoped and $\mathrm{Al}$-doped $\mathrm{ZnO}$ thin films deposited on glass substrate by spray pyrolysis, Materiel Chemistry and Physics, 2004, 83, 1, 43.
29. K. Schulzea, B. Maennig, and K. Leo, Organic solar cells on indium tin oxide and aluminum doped zinc oxide anodes, Appl. Phys. Lett., 2007, 91, 073521.

30. K. Iwata, T.Sakemi, A. Yamada, P. Fons, K. Awai, T. Yamamoto, S. Shirakata, K. Matsubara, H. Tampo, K. Sakurai, S. Ishizuka, S. Niki, Improvement of ZnO TCO film growth for photovoltaic devices by reactive plasma deposition (RPD), Thin Solid Films, 2005, 480-481, 1, 199-203.

31. BJ. Lu, NH. Sun, MW. Li, HZ. Dong, Nickle Oxide Based Bulk Heterojunction Flexible Solar Cells, Advanced Materials Research, 2012, 512-515, 109-112. 